\title{
„Generation Greta" - Herausforderungen für Religionsunterricht und Schule im Kontext eines Whole Institution Approach (WIA)
}

\author{
Simone Birkel \\ Katholische Universität Eichstätt-Ingolstadt
}

Kontakt: simone.birkel@ku.de

eingereicht: 30.05.2021; überarbeitet: 19.08.2021; angenommen: 24.09.2021

\begin{abstract}
Zusammenfassung: Weltweit hat sich eine Jugendbewegung rund um die Identifikationsfigur Greta Thunberg etabliert, die engagiert und durchsetzungsstark eine nachhaltige Lebensweise einfordert. Ihr Widerstand gegen traditionelle Strukturen fordert auch Verantwortliche für religiöse Bildung heraus, nach alternativen und zukunftsfähigen Wegen zu suchen, die den Religionsunterricht sinnstiftend und zielführend in den Bildungsdiskurs für Nachhaltige Entwicklung einbindet. Ausgehend von der Werteprägung der Generation Z und dem ganzheitlichen Ansatz eines Whole Institution Approachs (WIA) werden Bedingungen religiöser Bildung diskutiert, die klassische Unterrichtsmuster sprengen. Chancen bieten hier reformpädagogische Elemente, wie der Vernetzte Unterricht oder die Freie Stillarbeit, die insbesondere in kirchlichen Schulen der Marchtaler-Plan-Ausrichtung bereits erprobt werden.

Schlagwörter: Generation Greta, Religiöse Bildung für nachhaltige Entwicklung, Whole Institution Approach, Reformpädagogik

Abstract: Worldwide, a youth movement has been established around the figure of Greta Thunberg, who demands
a sustainable way of life in a committed and assertive way. The resistance to traditional structures challenges those
responsible for religious education to look for alternative and sustainable ways of integrating religious education
in a meaningful and purposeful way into the educational discourse on sustainable development. Based on the val-
ues of Generation $\mathrm{Z}$ and the holistic approach of a Whole Institution Approach (WIA), this article discusses condi-
tions of religious education that go beyond classical teaching patterns. Opportunities are presented by elements of
progressive education, such as interdisciplinary teaching or forms of independent work, methods which are al-
ready being tested, particularly in church schools with an orientation to the Marchtaler Plan.
Keywords: Generation Greta, Religious education for sustainable development, Whole Institution Approach, Pro-
gressive Education
\end{abstract}

Die Generation Z wird soziologisch öffentlichkeitswirksam auch als „Generation Greta" bezeichnet (Hurrelmann \& Albrecht, 2020), was hier - unter Berücksichtigung der damit verbundenen Problematik - übernommen wird. Wie tickt diese Generation, was ist ihr wichtig und welche Kennzeichen treffen auf sie zu? Was erwartet sie von Institutionen wie den Kirchen? Ist die Religionspädagogik als wissenschaftliche Disziplin auf Zukunft hin gesehen überhaupt anschlussfähig und könnte sie dieser Generation überhaupt etwas bieten?

Ein ganzheitlicher Ansatz, der in der BNE-Diskussion unter dem Label Whole Institution Approach (WIA) geführt wird, hat sich als möglicher Anknüpfungspunkt für eine zukunftsfähige Ausgestaltung von Ausbildungsorten herauskristallisiert. Dieser wird allerdings generell in der wissenschaftlichen Diskussion und explizit in der religionspädagogischen Forschung noch wenig diskutiert. Der Beitrag geht neben den oben erwähnten Aspekten der Frage nach, wie sich der WIA-Ansatz auf die Ausbildungsbereiche und insbesondere auf mögliche Veränderungen auf den Religionsunterricht (RU) auswirken könn- 
te. Was muss geschehen, damit religiöse Bildung im Diskurs einer BNE als eigene Stimme in einem sozialökologischen Transformationsprozess wahrgenommen werden kann? Und wie könnte eine mögliche Konkretisierung im Schulalltag aussehen?

\section{Generation Greta - Wer sie ist und was sie will}

Bis vor Kurzem war einiges nicht vorstellbar. Auch nicht, dass eine 15-jährige Schülerin eine globale Massenbewegung auslösen könnte und damit ihre Generation prägen würde. Der Soziologe und Generationenforscher Klaus Hurrelmann und der Journalist Erik Albrecht beschreiben die Generation Z, also die Menschen, die ab 2000 geboren wurden, in ihrem gleichnamigen Buch als „Generation Greta“ (Hurrelmann \& Albrecht, 2020). Hier ist gleich vorweg anzumerken, dass bei Generationenbeschreibungen immer nur Trends aufgezeigt werden. Junge Menschen wehren sich beispielsweise gegen die Vorstellung, dass sie nach Greta Thunberg benannt würden. Sie argumentieren, dass dies eine Zuschreibung von „alten weißen Männern“, eben jener beiden Autoren sei. Außerdem gibt es bei Menschen aus der Generation $\mathrm{Z}$ auch solche, die nicht in die Kategorie der gut gebildeten Klimaaktivist*innen passen wollen oder passen (Hoffmann, 2020, S. 19). Jenseits der Bezeichnung lassen sich für diese Generation jedoch Gemeinsamkeiten feststellen, wie verschiedene Jugendstudien bestätigen (Calmbach et al., 2020; Hoffmann, 2020; Shell Deutschland, 2019).

Ein kennzeichnendes Thema ist die Sorge einer Bedrohung durch den Klimawandel. Dies treibt sie bekanntlich ja auf die Straße. Sie finden neue Protestformen, einen Schulstreik gab es bis zum 20. August 2018 (Tag an dem Greta zum ersten Mal streikte) in dieser Form nicht. Die Jugendlichen organisieren sich über soziale Medien. Fridays-for-Future-Demonstrationen werden i. d. R. über WhatsApp-Gruppen geplant. Instagram ist das Medium der Wissensweitergabe und der Selbstpräsentation. Die jungen Menschen beherrschen die Inszenierung in den sozialen Medien. Die Generation Greta ist pragmatisch (Calmbach et al., 2020, S. 20) und sucht den Schulterschluss mit der Generation ihrer Eltern (Hurrelmann \& Albrecht, 2020, S. 46). Es kommt zu vielfältiger Allianzenbildung (Hurrelmann \& Albrecht, 2020, S. 59). So formierten sich Parents for Future, Scientists for Future, etc. in Windeseile. Das Wissen wird geteilt und allen zur Verfügung gestellt. Die jungen Menschen lesen beispielsweise die Nachhaltigkeitswissenschaftlerin Maja Göpel, die mit ihrem Besteller dazu einlädt, „,[u]nsere Welt neu [zu] denken“ (Göpel, 2020). Weitere prominente Unterstützer der Bewegung sind der Postwachstumsökonom Niko Paech und der Soziologe Hartmut Rosa (Werkstatt Zukunft, 2016). Auch in der pastoraltheologischen Diskussion wird gerne, wenn es darum geht, Leben in Fülle zu suchen, auf das Duo Paech \& Rosa rekurriert (Gehringer, 2017).

Politisch steht die Generation Greta für eine nachhaltige, transparente, partizipative, gendergerechte und diverse Lebensweise. Diskriminierung von Minderheiten verurteilen viele junge Menschen genauso wie gängige Mainstream-Berichterstattung über etablierte Medienformate. Das Onlinemagazin Rubikon, YouTube-Kanäle wie der von Esra Karakaya oder der Podcast „1,5 Grad“ mit Luisa Neubauer sind Beispiele ihrer Kommunikationsorgane. Junge und gebildete Frauen sind häufig Leitfiguren, wie Greta Thunberg, Esra Karakaya oder Luisa Neubauer prominent unter Beweis stellen. Auch bei lokalen Fridays-for-Future-Veranstaltungen sind es häufig junge Frauen, die an der Spitze der Bewegung stehen und das Wort ergreifen.

Eine ihrer wichtigsten Eigenschaften, die sich selbst zuschreiben ist Toleranz (Hurrelmann \& Albrecht, 2020, S. 78), auch die Shell-Jugendstudie von 2019 kommt zu dem Schluss: „Toleranz bleibt Markenzeichen" (Shell Deutschland, 2019, S. 86). Es ist immer wieder beeindruckend zu erleben, wie "vernünftig“ Jugendliche beispielsweise bei Demonstrationen die staatlichen Vorgaben beachten und umsetzen. Auch ein Zeichen dieser friedlichen Revolution ist, Andersdenkenden denselben Respekt zukommen zu lassen, den sie auch gegenüber sich selbst erwarten. 
Von etablierten Organisationen wie den Kirchen erwarten die postmateriell ausgerichteten Jugendlichen der Generation Greta erstmal gar nichts (Calmbach et al, 2020, S. 918). Diesbezüglich sind die jungen Menschen eher skeptisch, wenn nicht sogar misstrauisch, wiewohl es zu einzelnen lokalen Kooperationen kommt. Diese Skepsis lässt die Frage aufkommen, ob die Religionspädagogik respektive die Theologie für diese Generation überhaupt etwas zu bieten hätte. Diese Frage wird weiter unten aufgenommen werden, zunächst wird auf den für die Generation Greta relevanten Ansatz eines Whole Institution Approachs (WIA), eingegangen. Durch eine konsequente Verfolgung dieses Ansatzes werden nämlich genau die Forderungen einer ganzheitlichen und damit überzeugenden Bildung für nachhaltigen Entwicklung (BNE) in den Blick genommen.

\section{Charakteristika eines Whole Institution Approach (WIA)}

In der BNE-Diskussion besteht weitgehend Einigkeit darüber, dass Lernen am besten in geeigneten Umgebungen gelingen kann (Bundesministerium für Bildung und Forschung, o. J.). Verantwortung für die Mitwelt kann nicht einfach auswendig gelernt oder eingetrichtert werden. Um grundlegende Verhaltensänderungen zu erzielen, reicht es nicht, einmalig ein Nachhaltigkeitsprojekt durchzuführen oder Greta Thunberg in einer doppelstündigen Einheit als Prophetin des Klimaschutzes (Trömper \& Plagentz, 2019) zu behandeln. In der BNE-Diskussion wird deshalb immer wieder auf einen ganzheitlichen Ansatz, den sog. Whole Institution Approach (WIA) verwiesen. Bei der Suche nach Fachliteratur zum WIA erscheint in den gängigen wissenschaftlichen Datenbanken jedoch auf den ersten Blick sehr wenig, obwohl einzelne Untersuchungen dazu schon seit mehr als 15 Jahren auf dem Markt sind. Henderson und Tilbury geben beispielsweise für Australien einen Überblick und gehen auch auf die Wurzeln des WIA ein (Henderson \& Tilbury, 2004, S. 11). Allgemein gesehen leistet ein auf Interdisziplinarität und kollaborativer Zusammenarbeit basierender Ansatz, der auch unter dem Begriff des Whole Systems Design (WSD) erörtert wird, einen wesentlichen Beitrag zur Transformation von Bildungsorten (Pittmann, 2004). Auf einschlägigen Internetseiten wird in der Regel auf das BNE-Portal verwiesen oder sehr allgemein definiert: „,Bildung muss im Sinne des Whole Institution Approach (WIA), also als ganzheitlicher Ansatz, die sozialen, ökologischen und ökonomischen Dimensionen von Nachhaltigkeit vermitteln und analogen sowie digitalen Austausch ermöglichen. Dazu braucht es eine gerechte, abwechslungsreiche und zielgruppenorientierte Bildung“ (Nationale Plattform BNE, 2020). Durch den WIA, der als Schwerpunkt im UNESCO Weltaktionsprogramm BNE verankert ist, soll eine weltweite Transformation von Lehr- und Lernumgebungen angezielt werden (Nationale Plattform BNE, 2020).

Bei der Beschreibung dieser ganzheitlichen Betrachtungsweise werden interdisziplinär die Erkenntnisse der Bezugswissenschaften Pädagogik, Psychologie, Soziologie, Ökonomie, Architektur oder auch Transformationswissenschaften aufgenommen. Es wird davon ausgegangen, dass gerade auch bei der Bewältigung der durch die Pandemie hervorgerufene Krise im Bildungssystem die jeweiligen Lernorte von Kita über Schule und Universitäten gesamtheitlich im Sinne eines WIA gedacht werden müssen (BMBF, 2020, S. 2).

Es stehen also nicht allein die einzeln zu vermittelnden nachhaltigen Inhalte im Fokus, sondern die gesamte Einrichtung sollte sich idealerweise an Nachhaltigkeit ausrichten. Dazu gehören beispielsweise die Anpassung des Methodenrepertoires, die räumliche und verwaltungstechnische Ausstattung, eine an Nachhaltigkeit orientierte Beschaffung und Verpflegung, die Weiterbildung der Mitarbeitenden inklusive des Verwaltungspersonals sowie Fragen der Mobilität und der Zusammenarbeit mit außerschulischen Institutionen.

Die deutsche Übersetzung "ganzheitlicher BNE-Ansatz" greift einerseits verschiedene Prämissen reformpädagogischer Ansätze auf, was wiederum auf eine vielfältige Blickrichtung schließen lässt. Anderseits verkürzt das deutsche Wort „Ansatz“ den im Englischen mehrdimensional gebrauchten Begriff von approach. Dieser kann zwar durchaus als „Ansatz“ übersetzt werden, wird aber je nach Bedeutung 
auch als "Haltung", „Herangehensweise“, "Methode“ oder „Vorgehen“ verwendet und bringt so die Multiperspektivität des Anliegens besser zur Geltung, weshalb hier an der englischen Begrifflichkeit festgehalten wird.

Einen ersten optischen Überblick über die Bereiche des WIA in der Schule liefert die Grafik von Ingrid Hemmer und Ina Limmer. Er benennt die einzelnen Aspekte des WIA wie Unterricht, Betrieb, Schulkultur und Kooperationen.

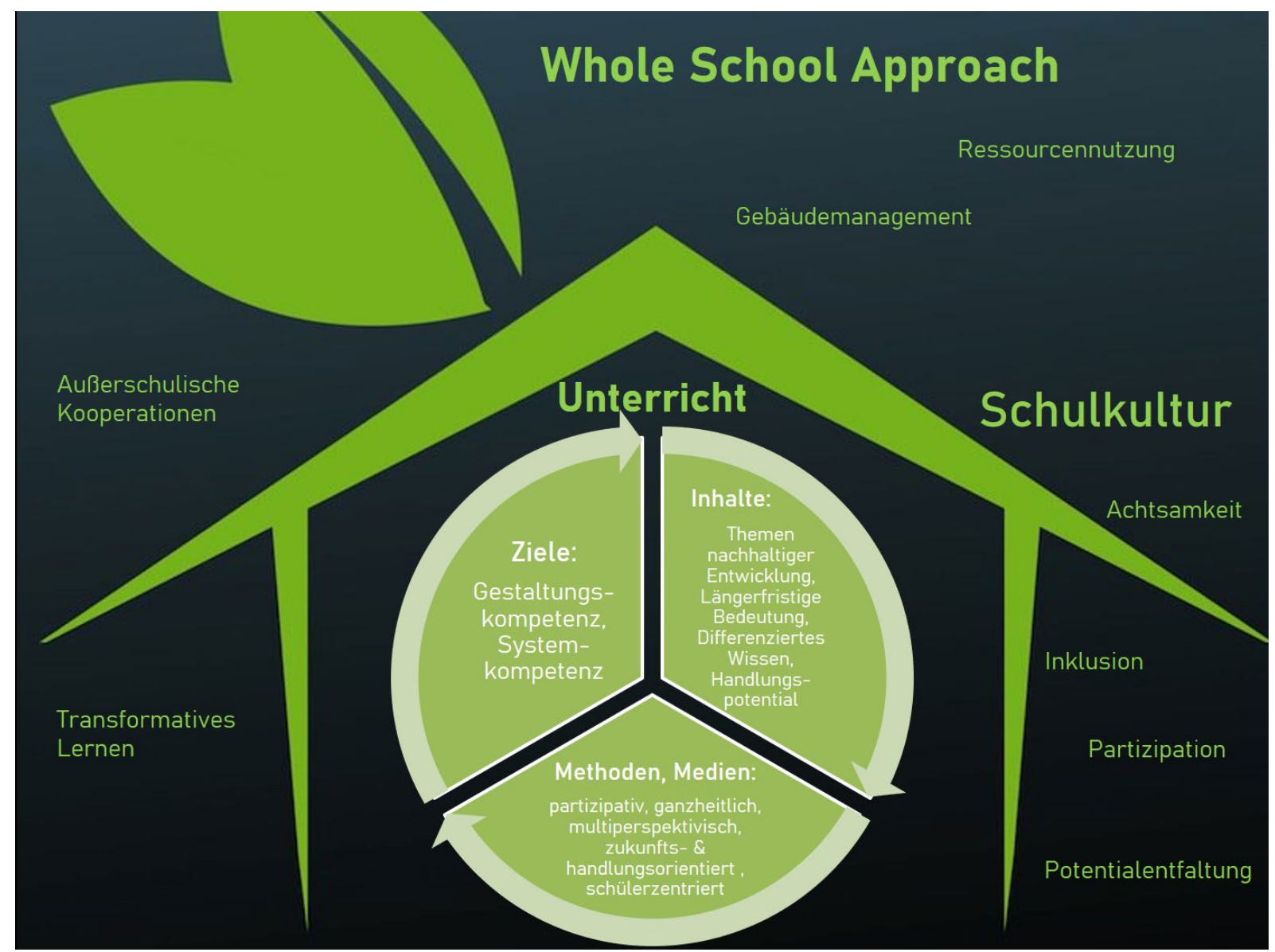

Abb. 1: Whole school approach (aktualisierte Version von Hemmer \& Limmer, 2021)

Zunächst steht in der Schule natürlich der Unterricht im Mittelpunkt. Sowohl Inhalte als auch Ziele und Methoden sollten so gewählt werden, dass auf ein Handeln in komplexen Systemen vorbereitet wird. Wichtig ist dabei auch die Ausbildung einer Schulkultur, die Partizipation, Transparenz, Achtsamkeit und die individuellen Potentiale der Schüler*innen oder Studierenden fördert. Das sind auch die anfangs erwähnten Anliegen der "Generation Greta“.

Ein wichtiger Aspekt von Institutionen, die einen WIA verfolgen, ist das damit innewohnende transformative Potential. Ulrike Lerche (2020) weist darauf hin, dass durch das konsequente Durchbuchstabieren von Nachhaltigkeit auf allen Ebenen Institutionen den Wandel einleiten: „Sie werden selbst zu Akteur/innen des gesellschaftlichen Wandels und tragen entscheidend zum Ausbau nachhaltiger (Wirtschafts-)Strukturen bei. Von ihren Mitarbeitenden und den Teilnehmenden werden nachhaltige Institutionen als authentisch und positives Vorbild wahrgenommen. Gleichzeitig eröffnen sie einen Erprobungsraum für nachhaltiges Arbeiten. Transparenz über das institutionelle Handeln, Partizipation und Handlungsorientierung spielen hier zusammen, wirken motivierend und inspirierend. Dies erhöht die Überzeugungskraft für alle Beteiligten - für die Adressat/innen von Bildungsangeboten, für Mitarbeitende, Geschäftsführung, Kooperationspartner/innen - nachhaltig zu handeln“ (Buchbesch, Lerche \& Niebling, 2020). 
Es geht also bei einem ganzheitlich ausgelegten Nachhaltigkeitsdiskurs auch um die Ausbildung von Haltungen. Wertschätzung, Offenheit, Rücksicht, Achtsamkeit etc. können nur weitergegeben werden, wenn sie selbst erfahren werden. Und genau in diesem Praxisfeld muss sich auch die religiöse Bildung bewähren. Ihr ureigenstes Anliegen ist es von jeher, „die großen Fragen des Menschseins und der Menschlichkeit: die Fragen nach Glück und Leid, Liebe und Ablehnung, Gelingen und Scheitern, Werden und Vergehen, Endlichkeit und Unendlichkeit, Kontingenz und Sinn“ (Kropač, 2019, S. 41) in den Blick zu nehmen. Junge Menschen spüren sehr genau, wer von den Lehrer*innen authentisch lebt oder wer nur ein Thema behandelt, das eben gerade in die Tages- oder Schulpolitik passt. Insbesondere von Religionslehrer*innen wird aufgrund der fachlich vertretenen Werteorientierung erwartet, dass ein $\mathrm{Zu}$ sammenhang zwischen persönlichem Lebensstil und schöpfungsverantwortlichen Handeln erkennbar ist.

\section{Religiöse Bildung und nachhaltige Entwicklung - neuer Wein in alten Schläuchen?}

Nachdem wenige engagierte Religionspädagog*innen schon vor ca. 20 Jahren dem Thema Nachhaltigkeit religionspädagogische Relevanz zusprachen (Birkel, 2002; Hisch, 2005; Reis, 2003), hat aktuell das Thema Nachhaltigkeit innerhalb der Religionspädagogik (endlich) Fahrt aufgenommen (Bederna, 2019; Bederna, 2020; Gärtner, 2020). Nicht zuletzt die Proteste und Anfragen der „Generation Greta“ haben Wissenschaftler*innen im Fach Religionspädagogik zum Schreiben bewegt, was sich beispielsweise in dem Themenheft „Nachhaltiges Lernen“ des Österreichischen Religionspädagogisches Forums (2/2020) und des vorliegenden Themenheftes der Religionspädagogischen Beiträge niederschlägt. Das Thema scheint somit in der Mitte der deutschsprachigen Religionspädagogik angekommen zu sein und endlich beginnt - unterstützt durch die Greta-Bewegung - eine vertiefte religionspädagogische Reflexion darüber, welchen Beitrag die Religionspädagogik im Diskurs hinsichtlich einer Transformation zu einer nachhaltigen Gesellschaft liefern kann.

Als mögliche Inhalte einer religiösen Bildung im Kontext von Nachhaltigkeit wurden und werden insbesondere die Verantwortung für die Schöpfung und ethisches Lernen im Sinne eines guten Lebens für alle definiert (Birkel, 2016, S. 8-13; Bederna, 2021, S. 328-331). Auch die zu erwerbenden Kompetenzen in diesem Bereich sind im Anschluss an Gerhard de Haan definiert (Bederna, 2019, S. 226-229). Grundsätzlich ist aber zu fragen, ob nicht religiöse Bildung im Kontext von Nachhaltigkeit zu sehr in klassischen Settings verhaftet bleibt. Zu überprüfen wäre, ob Menschen der Generation Greta damit etwas anfangen können, Klagemauern für Opfer der Klimakrise errichten, Klima-Bußgottesdienste durchzuführen oder subversive Aktionen wie Autos mit Aufklebern im Stile von Zigaretten-Warnhinweisen zu versehen (Bederna, 2021, S. 331). Ist es zielführend, im Religionsunterricht Konsumdiskussionen über den ökologischen Fußabdruck zu führen, die dann im Geografieunterricht wiederholt werden? Zwar spricht Bederna auch davon, die Strukturen im Großen zu reformieren, aber ob eine „Überarbeitung der Schulordnung unter Nachhaltigkeitskriterien“ (Bederna, 2021, S. 331) tatsächlich die notwendige Wende hin zu einer sozial-ökologischen Transformation in den Schulen herbeiführen wird, bleibt angesichts der Vielzahl an nicht umgesetzter Verordnungen zu bezweifeln. Hier ist auf die im Zuge der Nachhaltigkeitsdiskussion immer wieder genannte ganzheitliche Umstrukturierung des Lernortes Schule zu verweisen. Diese wird unter dem Stichwort WIA zwar oft proklamiert, es finden sich aber im schulischen Bereich (noch) zu wenig gelungene Beispiele, wie dieser konkret angegangen werden kann.

Wie lässt sich der WIA konkret an den Schulen durchbuchstabieren und in die Praxis umsetzen? Während im Bereich von kirchlichen Bildungshäusern schon erste Erfolge sichtbar sind (Birkel, 2020, S. 3f.), haben aktuell einzelne kirchliche Schulträger begonnen, sich systematisch dem Thema zu stellen (Erzdiözese München und Freising, 2020). Es gibt außerdem bestehende Schulkonzepte, die sich sehr gut dafür eignen, Nachhaltigkeit und WIA als Anliegen in ihre jeweilige Schulorganisation zu integrieren. Es ist dabei wenig verwunderlich, dass es sich bei diesen Konzepten um Modelle handelt, die von Haus 
auf ganzheitlich und reformpädagogisch ausgerichtet sind (Hisch, 2008). In der kirchlichen Bildungslandschaft sind dies beispielsweise Schulen, die nach der Marchtaler-Plan-Pädagogik arbeiten. Diese spezifische reformpädagogisch orientierte Schulvariante ist vorwiegend an katholischen Schulen in Süddeutschland verbreitet und wäre ein eigenes Thema für sich. Ich verweise hier auf den 2019 vorgelegten Band „Hoffnungsraum Schule“. Dort werden Perspektiven für eine profilierte Schulentwicklung ausführlich vorgestellt (Bieberstein, Nothaft \& Staudigl, 2019). Besonderes Augenmerk verdient der sog. Vernetzte Unterricht (VU). Er ist kompetenzorientiert angelegt und spielt eine entscheidende Rolle im Hinblick auf eine ganzheitliche und gesamtvernetzte Betrachtungsweise (Schleier, 2019), wie unten noch exemplarisch ausgeführt wird.

Insgesamt ist die Umsetzung eines WIA je nach Art und Ausrichtung der Bildungsinstitution sehr unterschiedlich, was hier im Einzelnen nicht weiter vertieft werden kann. Einen gemeinsamen Nenner bilden jedoch die folgenden Bereiche (Buckbesch, Lerche \& Niebling, 2020), die insbesondere auch für Schulen relevant sind:

Zunächst gilt es, Nachhaltigkeit als Teil des Alltagshandeln in der Institution zu verankern. Je nach Bildungsinstitution werden Kerngeschäfte wie Unterricht, Lehre, Forschung, gemeinsame Aktionen etc. mit nachhaltigen Inhalten bestückt.

Daneben sind die Bereiche Bewirtschaftung und Betrieb in den Blick zu nehmen. Dies umfasst beispielsweise die Bereiche Nachhaltigkeit in Beschaffung und Ausstattung, Verpflegung, Nutzung der Außenbzw. Dachflächen bis hin zur Frage der Mobilitätsgestaltung bei Anfahrt, Dienstreisen, Ausflügen oder Klassenfahrten.

Maßgeblich entscheidend für eine ganzheitliche Transformation sind auch die Bereiche Mitarbeiter*innenführung und Weiterbildungsmaßnahmen des gesamten Personals. Alle, Lehrkräfte, Verwaltungsangestellte, Gebäudetechniker*innen werden in den Prozess miteinbezogen. Religionslehrer*innen sind dabei in den Prozess eingebunden wie alle anderen Lehrkräfte auch. Für Schulen spielt auch die Einbindung der Eltern eine wichtige Rolle. Im Idealfall wird aus den verschiedenen Bereichen eine Steuerungs- oder Lenkungsgruppe gebildet, die den Transformationsprozess mitbestimmt. Aus diesem Grund ist auch die jeweilige Organisations- und Leitungskultur von Bedeutung, die von Partizipativität und Transparenz geprägt sein sollte. Die Entwicklung eines Leitbildes sowie die Mitarbeit am jeweiligen Profil sollte für alle möglich und auch selbstverständlich sein.

Entscheidend ist dabei auch die interne und externe Kommunikation. Erstere kann über institutionsübergreifende Nachhaltigkeitstage, Infomails, partizipative Events und Infoboards im Intranet oder/und an stark frequentierten Orten der Institution gewährleistet werden. Auch die Öffentlichkeitsarbeit nach auBen spielt eine wichtige Rolle, so dass die erreichten Fortschritte in Sachen Nachhaltigkeit i. d. R. durch Nachhaltigkeitsberichte, Homepages und regionale und überregionale Pressebeiträge kommuniziert werden. Gegenseitig voneinander zu wissen, erleichtert auch den Bereich der regionalen und überregionalen Kooperationen mit Engagierten in NGOs, Politik, Gesellschaft und Glaubensgemeinschaften. Oft ergeben sich gemeinsame Interessen wie beispielsweise im Bereich der dezentralen Energieversorgung oder im öffentlichen Personennahverkehr, die Energien für eine nachhaltige Transformation bündeln.

\section{Die Rolle der Leitung}

Wiederholt wird darauf hingewiesen, welche entscheidende Rolle die Leitung bei der Implementierung eines gesamtinstitutionellen Ansatzes innehat. Explizit haben Ulrich Müller und Armin Lude (2019, S. 1-34) auf die Rolle der Schulleitung bei einer gelingenden BNE hingewiesen und BNE als Führungsaufgabe von Schulleitungen durchbuchstabiert. Dabei weisen sie darauf hin, dass entscheidende Ziele von BNE, nämlich der Erwerb von Gestaltungs- und Handlungskompetenz, nur dann erreicht werden können, wenn durch Schulleitung eine entsprechende Unterstützung geleistet wird. Die Rolle und Aufgabe 
der Leitung beim Transformationsprozess ist maßgeblich, kann aber nur dann voll entfaltet werden, wenn ein breites Unterstützer*innenfeld vorhanden ist. So ist es eine zentrale Aufgabe der Leitung, den Nachhaltigkeitsprozess anzustoßen und mit entsprechenden Maßnahmen zu forcieren (Müller \& Lude, 2019, S. 10). Dabei ist bei der Schulleitung ein gewisses Maß an Überzeugung und auch eine entsprechende Haltung in Sachen Nachhaltigkeit von Nöten, um überzeugend und glaubwürdig wirken zu können. Müller und Lude sprechen hier in Anlehnung an Rolf Dubs von "transformationales Leadership“ (Müller \& Lude, 2019, S. 10), welches es ermöglicht, Prozesse der nachhaltigen Schulentwicklung zu steuern. Hierzu gehören neben der Fähigkeit der Kommunikation der eigenen Vision und Wertvorstellungen auch die Förderung der Inspiration und Identifikation der Mitarbeitenden mit der Institution sowie das Wahrnehmen der individuellen Bedürfnisse der Mitarbeitenden. Auch gilt es die Kreativität und den Ideenreichtum aller Beteiligten zu fördern, um dadurch als Leitung auch selbst wieder von neuen Sichtweisen inspiriert zu werden (Müller \& Lude, 2019, S. 10f.).

\section{Der Handlungsspielraum der Lehrer*innen}

Der Lehrberuf gehört zu einer anstrengenden und belastenden Tätigkeit. Scheuch, Haufe \& Seibt (2015) stellen heraus, dass psychische und psychosomatische Erkrankungen bei Lehrer*innen häufiger festgestellt werden als bei anderen Erwerbstätigen. Diagnostiziert wurden beispielsweise vermehrte Anteile von „Erschöpfung und Müdigkeit, Kopfschmerzen, Angespanntheit, Antriebslosigkeit, Schlaf- und Konzentrationsstörungen, innere Unruhe oder erhöhte Reizbarkeit" (Scheuch et al., 2015, S. 349). Im Sinne eines WIA geht es hier auch darum, eine Gesundheitskultur an den Schulen zu entwickeln. Nicht nur Schüler*innen sind hinsichtlich ihres Stresslevels zu befragen, sondern auch Lehrer*innen und Schulleitungen sollten regelmäßig dazu aufgefordert werden, darüber Auskunft zu geben, wie sie ihre Arbeitsbelastung einschätzen. So entwickeln sie ein Gefühl für Selbstfürsorge und können gegenüber sich selbst sowie gegenüber Kolleg*innen und Schüler*innen eine Sensibilität für einen achtsamen Umgang entwickeln. Pausen, Ruhezeiten und Regenerationsmöglichkeiten können in Form von Ritualen oder räumlichen Voraussetzungen wie z. B. einen Raum der Stille oder individuelle Rückzugsmöglichkeiten im Schulgelände unterstützt werden. Manche Schulen haben solche Oasen-Orte längst im Angebot.

Wenn an den Schulen Partizipation und Teamarbeit möglich ist, teilen sich mehrere Lehrkräfte pädagogische, fachliche, sorgende oder administrative Tätigkeiten. Was in reformpädagogisch orientierten Schulen oder auch in skandinavischen Ländern erfolgreich eingeführt und längst eine Selbstverständlichkeit ist, sind entsprechend zusammengesetzte Teams aus teilweise unterschiedlichen Berufen in den jeweiligen Klassenverbänden. Dabei wird das Stresslevel der einzelnen Personen reduziert, und es eröffnet sich dadurch auch die Möglichkeit, sich bei belastenden Situationen auch zurücknehmen zu können. In dem Wissen, dass die Klasse auf jeden Fall betreut ist, ohne dass andere Kolleg*innen Überstunden machen müssen, fällt es bisweilen leichter, eigene Schwächen einzugestehen und sich dafür auch Hilfe zu holen.

Auch der Aspekt der partizipativen Mitgestaltung und der Gewährung von individuellen Freiräumen für Lehrer*innen ist bei der Transformation der Bildungslandschaft maßgeblich. Werden alle Beteiligten von Anfang an in Schulentwicklungsprozesse miteinbezogen, kann gemeinsam festgelegt werden, in welchen Lernfeldern individueller Entwicklungsbedarf besteht. Ein lebenslanges, inter- und intragenerationales Lernen wird auf diese Weise ermöglicht.

Supervision und systemisches Denken spielen beim WIA eine wichtige Rolle. Viele schulische Probleme unterliegen nicht oder wenig beinflussbaren Faktoren und sind nicht selten systemimmanent. Innere Kündigungen in Form von Rückzug in der Arbeitsmotivation oder minimaler Arbeitseinsatz können oftmals ein Selbstschutz von Lehrer*innen auf die hohen Anforderungen an den Lehrberuf im derzeitigen System sein. Die Ursachen können durch einen systemischen Blick auf die Dinge und das Angebot 
von Supervision und Begleitung ans Tageslicht gelangen. Pittmann (2004, S. 210) hat bereits früh auf den gesundheitsförderlichen Aspekt eines ganzheitlichen Ansatzes hingewiesen: „Design, implementation, and evaluation which are aligned with a vision of relational health and integrity honor a diversity of perspectives, hopes, and concerns. These can move us toward continuous personal, organizational, and cultural healing on a shared path of living sustainability." Engagierte, interessierte, zufriedene, zukunftsoffene und agile Lehrer*innen sind die entscheidenden Voraussetzungen dafür, um Kinder und Jugendliche dabei zu unterstützen, die Welt trotz Krisen zukunftsfähig zu gestalten. Kritisch reflektierte und pädagogisch versierte Lehrer*innen bieten auch für Jugendliche, die den Klimawandel leugnen oder die für Verschwörungserzählungen affin sind, das notwendige Reibungs- und Wachstumspotential.

\section{Was RU im Kontext eines WIA leisten kann}

Religionsunterricht, der im Verbund mit anderen Fächern organisiert wird, kann in vielerlei Hinsicht einen Beitrag für eine ganzheitliche Ausrichtung der Schule leisten. Zunächst ist RU oft von Haus aus ganzheitlich ausgerichtet. Rituale und liturgische Elemente sind im Sinne der ästhetischen Bildung von jeher Teil des RU. Biblische Geschichten können im Sinne des Storytelling Visionen von einer gerechten Welt entstehen lassen und eröffnen damit neue Ermöglichungsräume (Bieberstein, 2015, S. 352-355). Im Kontext der Reich-Gottes-Botschaft verweisen Christ*innen auf eine schöpfungsverantwortliche, partizipative, transparente und tolerante Lebensweise mit allen Mitgeschöpfen und treffen damit wiederum die Vorstellungen der "Generation Greta“. Diakonische Projekte, die im RU angestoßen werden, prägen nicht selten das Leben der Schulfamilie. Andererseits bleibt der RU noch viel zu oft in seinem Sonderstatus der Konfessionalität, kirchlicher Eigenheiten und wissenschaftlich nicht immer nachvollziehbaren lehramtlichen Aussagen verhaftet. Insbesondere junge Frauen der Generation Greta distanzieren sich aufgrund der fehlenden Gleichstellung von der Institution Kirche vor allem im katholischen Bereich. Ein Vernetzter Unterricht (VU), der beispielsweise in gemeinsamer Verantwortung mit Biologie und Erdkunde angelegt ist, kann solche Verengungen phasenweise sprengen. Im ersten VU-Themenkreis „Heimat" zu Beginn des Schuljahres an einer weiterführenden Schule wird beispielsweise deutlich, dass ausgehend von der regionalen Verortung der Schüler*innen zunächst geografische Fakten wichtig sind. Wo komme ich her, was trägt und prägt mich? Die Beschäftigung mit der eigenen Region und Herkunft ist aber auch hinsichtlich einer Selbstvergewisserung relevant. Wo sind meine Wurzeln, welche Einstellungen bzw. welches Mindset bringe ich mit? Explizit religiöse Inhalte, hier als „Orte und Spuren des Glaubens“ definiert, werden innerhalb des Gesamtthemas „Heimat“ behandelt. Die für die Schüler*innen notwendigen Identitätsfragen werden hier nicht losgelöst, sondern im Verbund mit der politischen Gemeinde, der Region und im Kontext von neuer Klassengemeinschaft erarbeitet.

Viele weitere bildungsrelevante Aspekte wie beispielsweise die Endlichkeit des Lebens, der Umgang mit Versagen, oder die Frage nach einem Sinnhorizont für gutes und authentisches Leben in einer von Wettbewerb geprägten Umgebung können von lang geprägten religiösen Trauerformen, religiöser Erfahrung mit Vulnerabilität oder resilienzerprobten religiösen Lebensform profitieren (Schneider, 2018, S. 18f.), wenn sie ganzheitlich, interdisziplinär und im Verbund der Fächer angegangen werden.

Eine Umgestaltung von Schule im Sinne eines Whole Institution Approach geht nicht von heute auf morgen. Sie ist ein Prozess, der Jahre dauert. Und sie geht auch nur, wenn alle Beteiligten und nicht nur einzelne Bereiche einbezogen werden. Es braucht dazu eine Steuerung und Begleitung. Es gibt bereits gelungene Beispiele, wie solche Ausbildungsstätten der Zukunft aussehen könnten (Rasfeld \& Breidenbach, 2014). Klar ist, und das führt uns die „Generation Greta“ vor Augen, es kann nicht so weitergehen wie bisher. Und das Gute daran ist: Wir können und dürfen die junge Generation in diesen Transformationsprozess mit einbeziehen. 


\section{Literaturverzeichnis}

Bederna, Katrin (2019). Every Day for Future. Theologie und religiöse Bildung für nachhaltige Entwicklung. Ostfildern: Matthias Grünewald.

Bederna, Katrin (2020). Art. Bildung für nachhaltige Entwicklung. Das wissenschaftlich-religionspädagogische Lexikon im Internet. https://doi.org/10.23768/wirelex.Bildung fr nachhaltige Entwicklung.$\underline{200572}$

Bederna, Katrin (2021). Didaktik religiöser Bildung für nachhaltige Entwicklung. In Ulrich Kropač \& Ulrich Riegel (Hg.), Handbuch Religionsdidaktik (S. 325-331). Stuttgart: Kohlhammer.

Bieberstein, Sabine (2015). Jesus und die Evangelien (Studiengang Theologie II,1). Zürich: Theologischer Verlag.

Bieberstein, Sabine; Nothaft, Peter \& Staudigl, Barbara (2019). Hoffnungsraum Schule. Perspektiven für eine profilierte Entwicklung katholischer Schulen. Ostfildern: Matthias Grünewald.

Birkel, Simone (2002). Zukunft wagen - ökologisch handeln. Grundlagen und Leitbilder kirchlich-ökologischer Bildung im Kontext nachhaltiger Entwicklung. (Dissertation, Katholische Universität Eichstätt-Ingolstadt)

Birkel, Simone (2016). Die Sorge für das gemeinsame Haus. Herausforderungen für die Religionspädagogik durch die Mitwelt-Enzyklika Laudatio sí. In Religionspädagogische Beiträge, 75, 5-13.

Birkel, Simone (2020). Nachhaltig Zukunft gestalten. Herausforderungen für kirchliche Bildungsakteur*innen. cúangel: magazin für missionarische pastoral, 1, 1-7.

Buckbesch, Mirja; Lerche, Ulrike \& Niebling, Lina (4. Mai 2020). Leben, was wir lehren - mit dem Whole Institution Approach Bildungseinrichtungen nachhaltiger gestalten. Abgerufen von https://www.wb-web.de/dossiers/nachhaltigkeit/folge-2-nachhaltigkeit-in-einrichtungen-der-erwachsenenbildung/lebenwas-wir-lehren.html [16.05.2021]

Bundesministerium für Bildung und Forschung (o. J.). Bildung für nachhaltige Entwicklung (BNE) als Weg als Weg aus der Krise. Eine Resolution der Nationalen Plattform BNE. Aufgerufen von https://www.bmbf.de/bmbf/shareddocs/downloads/files/resolution np bne-als-weg-aus-der-krise final.pdf? blob=publicationFile\&v $=1$ [16.05.2021]

Bundesministerium für Bildung und Forschung (o.J.). Whole Institution Approach - der ganzheitliche BNEAnsatz. Abgerufen von https://www.bne-portal.de/de/whole-institution-approach---der-ganzheitliche-bne-ansatz-1778.html [16.05.2021]

Calmbach, Marc; Flaig, Bodo; Edwards, James; Möller-Slawinski, Heide; Borchard, Inga \& Schleer, Christoph (2020). Sinus-Jugendstudie 2020 - Wie ticken Jugendliche? Lebenswelten von Jugendlichen im Alter von 14 bis 17 Jahren in Deutschland. Bonn: Bundeszentrale für politische Bildung.

Deutsche Shell Holding GmbH (Hg.) (2019). Jungend 2019 - 18. Shell Jugendstudie. Eine Generation meldet sich zu Wort. Weinheim: Beltz.

Deutsche UNESCO-Kommission (o. J.). Whole Insitituion Approach - der ganzheitliche BNE-Ansatz. Abgerufen von https://www.unesco.de/node/6100 [16.05.2021]

Erzdiözese München und Freising (2020). Die Schulen der Erzdiözese München und Freising. Schöpfungsverantwortung. Abgerufen von https://schulen.katholisch.de/Portals/0/PDF/Schulen der Erzdio\%CC\%88zese Scho\%CC\%88pfungsverantwortung web.pdf?ver=2021-02-12-133120-003\&timestamp$=1613137737270$ [16.05.2021]

Gärtner, Claudia (2020). Klima, Corona und das Christentum. Religiöse Bildung für nachhaltige Entwicklung in einer verwundeten Welt. Bielefeld: transcript.

Gehringer, Dominik (13. November 2017). Postwachstumspastoral? Ideen von Niko Paech und Hartmut Rosa. Feinschwarz - Theologisches Feuilleton. Abgerufen von https://www.feinschwarz.net/wege-indie-postwachstumspastoral-ideen-von-niko-paech-und-hartmut-rosa/ [24.11.2020]

Göpel, Maja (2020). Unsere Welt neu denken: Eine Einladung (9. Auflage). Berlin: Ullstein.

Hemmer, Ingrid \& Limmer, Ina (2021). Wie können wir unsere Schulen nachhaltiger gestalten? Ein 
Whole Institution Approach. In Gabriele Obermaier, Manfred Miosga, Gabriele Schrüfer \& Kati Barthmann (Hg.), Nachhaltigkeit. Bayreuth: Naturwissenschaftliche Gesellschaft Bayreuth e. V. (in Druck).

Henderson, Kate \& Tilbury, Daniella (2004). Whole-school approaches to sustainability: An international review of whole-school sustainability programs. Report prepared by the Australian Research Institute in Education for Sustainability (ARIES) for The Department of the Environment and Heritage, Australian Government. https://aries.mq.edu.au/projects/whole school/files/international review.pdf

Hisch, Johann (2005). Das Thema Nachhaltigkeit als gemeinsame religionspädagogische Herausforderung. In Österreichisches Religionspädagogisches Forum, 15(1), 33-34.

Hisch, Johann (2008). PILGRIM-Schule. Ein Modell der Bildung für Nachhaltigkeit. In Diakonia, 39, 218-222.

Hoffmann, Elisabeth (2020). Generation friedliche Revolution - Jugend 2020. Berlin: Konrad-Adenauer-Stiftung. Abgerufen von https://www.kas.de/documents/252038/7995358/Generation+friedliche+Revolution+-+Jugend+2020.pdf/ca0d0cc4-2c87-0b4f-24cd-f2d438b78e5b?version=1.0\&t $=1601554417679$ [16.05.2021]

Hurrelmann, Klaus \& Albrecht, Erik (2020). Generation Greta. Was sie denkt, wie sie fühlt und warum das Klima erst der Anfang ist. Weinheim: Beltz.

Kropač, Ulrich (2019). Religion. Religiosität. Religionskultur. Ein Grundriss religiöser Bildung in der Schule. Stuttgart: Kohlhammer.

Müller, Ulich \& Lude, Armin (2019). Bildung für eine nachhaltige Entwicklung als Schulleitungsaufgabe. Schulleitung und Schulentwicklung, 1-34.

Pittmann, James (2004). Living Sustainably through Higher Education: A Whole Systems Design Approach to Organizational Change. In Peter B. Corcoran \& Arjen E.J. Wales (Eds.), Higher Education and the Challenge of Sustainability. Problematics, Promise, and Practice (pp. 199-212). Dordrecht: Kluwer Academic Publishers.

Rasfeld, Margret \& Breidenbach, Stephan (2014). Schulen im Aufbruch - Eine Anstiftung. München: Kösel.

Reis, Oliver (2003). Nachhaltigkeit - Ethik - Theologie. Eine theologische Beobachtung der Nachhaltigkeitsdebatte. Münster: LIT.

Scheuch, Klaus; Haufe, Eva \& Seibt, Reingard (2015). Teachers' health. Deutsches Ärzteblatt, 112, 347356. https://www.doi.org/10.3238/arztebl.2015.0347

Schleier, Bernd (2019). Marchtaler Plan. Vernetzter Unterricht. Das wissenschaftlich-religionspädagogische Lexikon im Internet. https://doi.org/10.23768/wirelex.Marchtaler Plan Vernetzter Unterricht.100231

Schneider, Martin (2018). Resilienz, Verwundbarkeit und Sicherheit. Was Theologie und Ethik zum Resilienzdiskurs beitragen können. zur debatte, 7, 17-20.

Trömper, Anke \& Plagentz, Achim (2019). „Ihr seid nicht erwachsen genug, die Wahrheit zu sagen." Greta Thunberg - eine Prophetin des Klimaschutzes? Ein Entwurf für die Sekundarstufe 1 und die Konfirmandenarbeit. Abgerufen von https://www.rpi-ekkw-ekhn.de/fileadmin/templates/rpi/normal/bilder/orte/fulda/UE Klimafasten Sek1 KonfiArbeit RPI EKKW EKHN.pdf [16.05.2021]

Werkstatt Zukunft (15. Juni 2016). Niko Paech und Hartmut Rosa im Gespräch, Wachstum \& Beschleunigung - Muss das sein? [Video]. YouTube. https://www.youtube.com/watch? $\mathrm{v}=\mathrm{ANhoK60aHP8 \& feature=-}$ $\underline{\text { emb } \log 0}$ 